\title{
Metachronous Osteosarcoma
}

National Cancer Institute

\section{Source}

National Cancer Institute. Metachronous Osteosarcoma. NCI Thesaurus. Code C38157.

A bone osteosarcoma that has metastasized to skeletal or extraskeletal sites. 